\title{
Effect of achieving blood pressure targets on the relative telomere length in hypertensive patients with and without type 2 diabetes mellitus
}

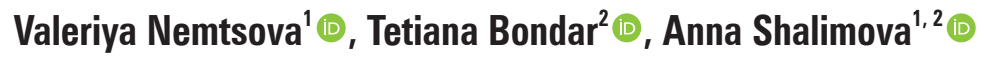 \\ ${ }^{1}$ Kharkiv National Medical University, Kharkiv, Ukraine \\ ${ }^{2}$ Government Institution 'L.T. Malaya Therapy National Institute of the National Academy of Medical Sciences of Ukraine, \\ Kharkiv, Ukraine
}

\begin{abstract}
Background. The role of relative telomere length (RTL) as a marker of cardiovascular prognosis and quality control of the disease course remains to be found out. The aim of the study was to determine the relationship between the relative blood leukocyte telomere length (RLTL), relative buccal epithelium cell telomere length (RBTL) and achieving blood pressure targets (BPTs) in hypertensive $(\mathrm{H})$ individuals with type 2 diabetes mellitus (T2DM) and without T2DM.

Material and methods. In 156 patients with stage II hypertension (96 of them had T2DM), carbohydrate metabolism parameters and blood pressure levels were evaluated. RLTL and RBTL were determined by a real time quantitative PCR.

Results. A combination of hypertension and T2DM was associated with significantly greater RLTL ( $p=0.009)$ and RBTL ( $p=0.001)$ compared with isolated hypertension. There was no convincing evidence of BPT influence on the change in the RLTL in isolated hypertension. Achieving BP targets was associated with a paradoxical shortening of RBTL. There was a significant shortening of RTL in hypertensive patients with T2DM when the BPTs were not reached. An analysis of variance revealed a significant influence of BPTs on the RBTL and RLTL.

Conclusion. Patients with a comorbid course of hypertension and T2DM had more pronounced decrease in the RTL. Target levels of blood pressure have a more significant effect on the RLT than the glycemic control in patients with concomitant T2DM. An additional determination of the RBTL enhances the diagnostic and prognostic power when evaluating the effectiveness of correcting cardiovascular risk factors including blood pressure.
\end{abstract}

Key words: hypertension; type 2 diabetes mellitus; relative telomere length; cardiovascular risk factors

$$
\text { Arterial Hypertens. 2020, vol. 24, no. 2, pages: 61-66 }
$$

DOI: $10.5603 /$ AH.a2019.0016

Address for correspondence: Anna Shalimova

Government Institution 'L.T. Malaya Therapy National Institute of the National Academy of Medical Sciences of Ukraine',

Malaya 2a av., 61039 Kharkiv, Ukraine; e-mail: anna.shalimova83@gmail.com 


\section{Introduction}

Telomeres are gene sequences present at chromosomal ends and are responsible for maintaining genome integrity. Telomere length decreases progressively with advancing age and thus is considered as a biomarker of chronological aging. This age-associated decrease in the length of telomere is linked to various age-associated diseases like diabetes, hypertension, Alzheimer's disease etc. [1]. There are studies which support that telomere length is associated with a number of chronic conditions including dyslipidemia, hypertension, atherosclerosis, and poor cardiovascular disease prognosis [2,3]. Furthermore, a number of studies indicate that telomere attrition is positively correlated with diabetes and diabetic complications $[4,5]$.

A number of studies on various samples show that the average telomere length of circulating leukocytes is shorter in hypertensive than in normotensive subjects [6]. The study shows that moreover, telomere length represents an independent risk factor for the development of coronary artery disease both in patients with hypertension and in subjects with normal blood pressure (BP) [6]. Modern studies of telomere length dynamics also show that aging and age-related diseases, in addition to synergistic effects, affect telomere length independently of one another.

Impact on modified risk factors, one the most significant among them being considered BP levels, is one of the strategic tasks of treating patients with the established diagnosis of cardiovascular disease, including arterial hypertension. Although classic risk factors have been found to predict the occurrence of hypertension, there is wide variation in disease manifestation in individuals who have the same risk profile. It is believed that telomere length can be considered as a marker of cardiovascular aging [7], but its role in cardiovascular prognosis and quality control of the disease course remains to be clarified.

Blood is commonly used to study telomere lengths, since peripheral blood leukocytes are an excellent object for studying the dynamics of telomeres. Many authors believe that the method for determining the telomere length in the peripheral blood can provide reliable data for determining the relative length of telomeres in other tissues [8] and shortening of telomeres in peripheral blood leukocytes can serve not only as a marker of aging, but also as an indicator of the systemic pathological stress. In recent years, more and more scientific papers have appeared, in which much attention is paid to the use of buccal epithelium as an alternative source of biological material for telomeric test due to the non-invasive pro- cedure of material sampling $[9,10]$. Nowadays, there is insufficient data on the length of telomeres, including that in the buccal epithelium, in the presence of the combined age-associated pathology, on the influence of various risk factors, primarily modifiable, on the relative length of telomeres, including that of the Ukrainian population. Data on the comparative characteristics of the relative telomere length (RTL) in the blood leukocytes and buccal epithelial cells in combined course of arterial hypertension and type 2 diabetes mellitus (T2DM), as the most frequently encountered combined pathologies among older age groups, and the effect of different levels of BP control on telomere length in this category of patients has not been found.

The aim of the study was to determine the impact of achieving BP targets on the relative telomere length of blood leukocytes and buccal epithelium in individuals suffering from hypertension, both in combination with T2DM and without T2DM.

\section{Material and methods}

The study included 156 patients (62 men and 94 women, the average age $-62.66 \pm 4.21$ years $)$, with hypertension stage II (disease duration of 10.2 \pm 3.7 years); 96 of them had T2DM (disease duration of $4.1 \pm 2.4$ years). All patients were divided in two groups: group $1-60$ patients with isolated hypertension $(n=60)$ and group $2-$ patients with hypertension and T2DM. The control group consisted of 22 volunteers - age- and gender-matched and without cardiovascular or endocrine diseases. For the patients' selection, the hypertension diagnostic criteria, in accordance with the ESC/ERS Guidelines (2013), were applied [11]. The diagnosis of T2DM has been established in compliance with the international recommendations of the American Diabetes Association and the European Association for the Study of Diabetes [12].

Against the background of dietary recommendations, all patients received basic therapy in accordance with international and national guidelines for the management of patients with the relevant pathology $[11,12]$. Therefore, stable antihypertensive therapy was received by all patients for at least 6 months prior to inclusion into the study with individually-selected doses using angiotensin-converting enzyme inhibitors or angiotensin II receptor blockers (ACEI/ARB), diuretics (indapamide); some patients received calcium antagonists (amlodipine or lercanidipine). As antidiabetic therapy, patients with T2DM received metformin in individually-selected 
doses ranging from 1000 to $2000 \mathrm{mg}$ per day, and 3 patients $(3.13 \%)$ additionally received a sodium glucose linked transporter-2 inhibitor (SGLT2) and 5 patients $(5.21 \%)$ - an agonist of glucagon-like peptide 1 (GLP 1). The study did not include patients with diabetes mellitus type 1 (and other endocrinological disorders), symptomatic hypertension, clinical signs of coronary heart disease or severe concomitant chronic diseases.

$\mathrm{BP}$ was recorded as the arithmetic mean, three measurements being carried out with the 2-minutes intervals in a sedentary position on the dominant hand.

Laboratory tests included the determination of fasting glucose concentration and glycosylated hemoglobin $\left(\mathrm{HbA}_{1 \mathrm{c}}\right)$ levels by standard conventional techniques.

Telomere length was measured on a CFX96 touch real-time polymerase chain reaction detection system (Bio-Rad Laboratories) in DNA isolated from blood leukocytes and buccal epithelium using the DNAsorb blood kit (Amplisens, RF). We used a modified monochrome multiplex quantitative polymerase chain reaction (PCR) method [13], as described previously [14]. The telomere length was calculated using the $\Delta \Delta$ Ct method [13].

Statistical data processing was carried out using the computer software IBM SPSS Statistics V. 21.0. To determine the nature of the data distribution, the Shapiro-Wilk criterion was used. When performing the statistical analysis, quantitative and qualitative variables were used. Qualitative data were presented as percentages; quantitative data - in the form of the mean and standard error $(\mathrm{M} \pm \mathrm{m})$. Testing the significance of differences between the two groups was carried out using the Student's t-test. The frequency of traits in the groups was compared using the Pearson $\chi^{2}$ test. The differences were statistically significant at $\mathrm{p}<0.05$. When studying the influence of risk factors on the telomere relative length, singlefactor analysis of variance was used. Testing the hypothesis of dispersion homogeneity was performed using the Levene's test.

The work was performed in compliance with the basic provisions of the World Medical Association (WMA) Declaration of Helsinki on ethical principles for medical research involving human subjects (1964-2000) and MOH of Ukraine Order No. 690 dated September 23, 2009. The study was approved by the Bioethics Commission at the Kharkiv National Medical University in accordance with the principles set forth in the Declaration of Helsinki.

\section{Results}

The levels of both systolic BP (SBP) and diastolic BP (DBP) in patients of groups 1 and 2 reliably differed from those in the control group (Tab. 1).

The presence of comorbid pathology was accompanied by higher SBP and DBP levels than in isolated hypertension; however, significant differences were only observed for SBP $(\mathrm{p}=0.012)$. The presence of hypertension, regardless of concomitant T2DM, was accompanied by a significant shortening of the RTL compared with the control group. The presence of T2DM was accompanied by an even greater shortening of telomere length, reaching reliable differences both in RBTL ( $p=0.001)$ and RLTL ( $p=0.009)$.

We assessed the impact of the achieved target BP according to ESC guidelines (2018) on the RTL [15]. Thus, despite the long-term antihypertensive therapy and permanent BP control, which was the criterion for inclusion in our study, the target SBP levels was not reach by 23 (38.33\%) patients in

Table 1. Comparative characteristics of carbohydrate indices, blood pressure levels and relative telomere length $(\mathrm{M} \pm \mathrm{m})$

\begin{tabular}{|l|c|c|c|c|}
\hline Index & Group1 $\mathbf{( n = 6 0 )}$ & Group 2 (n= 96) & Control (n= 22) & Significance (p) \\
\hline Fasting blood glucose [mmol/L] & $5.45 \pm 0.12$ & $8.90 \pm 0.50$ & $4.62 \pm 1.08$ & $\mathrm{p}_{1-2}<0.001$ \\
\hline $\mathrm{HbA}_{1 \mathrm{c}}(\%)$ & $6.13 \pm 0.11^{*}$ & $7.61 \pm 0.19^{*}$ & $4.74 \pm 1.10$ & $\begin{array}{c}\mathrm{p}_{1-2}<0.001 \\
{ }^{*} \mathrm{p}=0.001\end{array}$ \\
\hline SBP [mm Hg] & $138.95 \pm 2.83^{*}$ & $146.65 \pm 2.74^{*}$ & $125.05 \pm 3.86$ & $\begin{array}{c}\mathrm{p}_{1-2}=0.012 \\
{ }^{*} \mathrm{p}=0.001\end{array}$ \\
\hline DBP [mm Hg] & $86.71 \pm 1.62$ & $90.05 \pm 1.59^{*}$ & $78.19 \pm 7.01$ & ${ }^{*} \mathrm{p}<0.001$ \\
\hline $\begin{array}{l}\text { Relative blood leukocyte telomere } \\
\text { length (RLTL) [T/S] }\end{array}$ & $1.27 \pm 0.08$ & $0.97 \pm 0.08^{*}$ & $1.66 \pm 0.52$ & $\begin{array}{c}\mathrm{p}_{1-2}=0.009 \\
{ }^{*} \mathrm{p}=0.005\end{array}$ \\
\hline $\begin{array}{l}\text { Relative buccal epithelium cell } \\
\text { telomere length (RBTL) [T/S] }\end{array}$ & $1.26 \pm 0.08^{*}$ & $0.90 \pm 0.08^{*}$ & $2.33 \pm 0.51$ & $\begin{array}{l}\mathrm{p}_{1-2}=0.001 \\
{ }^{*} \mathrm{p}<0.001\end{array}$ \\
\hline
\end{tabular}

$\mathrm{HbA}_{1 \mathrm{c}}$ - glycosylated hemoglobin; SBP — systolic blood pressure; DBP — diastolic blood pressure; *The difference is significant compared with the control group; $\mathrm{p}_{1-2}$ - the difference is significant between group 1 and group 2 
Table 2. Comparative characteristics of relative telomere length in dependence of achieving blood pressure targets $(\mathrm{M} \pm \mathrm{m}$ )

\begin{tabular}{|l|c|c|c|c|c|c|c|c|}
\hline & \multicolumn{4}{|c|}{ Group1 (n= 60) } & \multicolumn{3}{c|}{ Group 2 (n = 96) } \\
\hline Index & \multicolumn{2}{|c|}{ RLTL [T/S] } & \multicolumn{2}{c|}{ RBTL [T/S] } & \multicolumn{2}{c|}{ RLTL [T/S] } & \multicolumn{2}{c|}{ RBTL [T/S] } \\
\hline $\mathbf{Z}$ & Yes & No & Yes & No & Yes & No & Yes & No \\
\hline SBP $[\mathrm{mm} \mathrm{Hg}]$ & $1.33 \pm 0.06$ & $1.20 \pm 0.08$ & $1.17 \pm 0.05$ & $1.45 \pm 0.06^{*}$ & $1.15 \pm 0.07$ & $0.86 \pm 0.06^{*}$ & $0.87 \pm 0.03$ & $0.84 \pm 0.08$ \\
\hline DBP [mm Hg] & $1.21 \pm 0.05$ & $1.34 \pm 0.03^{*}$ & $1.13 \pm 0.06$ & $1.36 \pm 0.04^{*}$ & $1.08 \pm 0.04$ & $0.90 \pm 0.07^{*}$ & $0.85 \pm 0.04$ & $0.83 \pm 0.05$ \\
\hline $\begin{array}{l}\text { SBP and DBP } \\
{[\mathrm{mm} \mathrm{Hg}]}\end{array}$ & $1.26 \pm 0.05$ & $1.21 \pm 0.02$ & $1.15 \pm 0.04$ & $1.32 \pm 0.08^{*}$ & $1.22 \pm 0.06$ & $0.88 \pm 0.04^{*}$ & $0.87 \pm 0.03$ & $0.86 \pm 0.06$ \\
\hline
\end{tabular}

RLTL — relative blood leukocyte telomere length; RBTL — relative buccal epithelium cell telomere length; SBP — systolic blood pressure; DBP — diastolic blood pressure; *The difference is significant compared with achieving target BP levels in appropriate subgroup and indicator

Table 3. Comparative characteristics of relative telomere length in dependence of achieving target levels of carbohydrate metabolism in patients with hypertension and type 2 diabetes mellitus $(\mathrm{M} \pm \mathrm{m})$

\begin{tabular}{|l|c|c|c|c|}
\hline \multicolumn{4}{|c|}{ Group 2 (n= 96) } & \multicolumn{2}{c|}{ RBTL [T/S] } \\
\hline Index & \multicolumn{2}{|c|}{ RLTL [T/S] } & Yes & No \\
\hline Presence of compensation & Yes & No & $0.95 \pm 0.04$ & $0.78 \pm 0.06^{*}$ \\
\hline Fasting glucose [mmol/L] & $0.83 \pm 0.06$ & $0.96 \pm 0.03$ & $1.03 \pm 0.03$ & $0.60 \pm 0.04^{*}$ \\
\hline $\mathrm{HbA}_{1 \mathrm{c}}(\%)$ & $0.82 \pm 0.03$ & $1.04 \pm 0.05^{*}$ & \\
\hline
\end{tabular}

RLTL — relative blood leukocyte telomere length; RBTL — relative buccal epithelium cell telomere length; *The difference is significant $(\mathrm{p}<0.05)$ compared with achieving target levels in appropriate subgroup and indicator

group 1 and by $57(59.37 \%)$ patients in group 2 . Thirty-five $(58.33 \%)$ patients in group 1 and 77 $(80.21 \%)$ patients in group 2 did not reach the target DBP level. The target levels of both SBP and DBP were not achieved by 35 (58.33\%) patients in group 1 and by 57 (59.38\%) patients in group 2. There was no convincing evidence obtained in patients with isolated hypertension of the influence of reaching target BP levels (both SBP and DBP) on the change in the RLTL. Blood pressure targets were accompanied by a paradoxical shortening of RBTL (Tab. 2).

The presence of concomitant T2DM is accompanied by a significant shortening of the RTL when the target levels of both SBP and DBP are not reached, which is a significant fact confirming the importance of BP control in T2DM (RLTL when the target levels of SBP are reached $-1.15 \pm 0.07$, and when the target levels of SBP are not reached $-0.86 \pm 0.06, \mathrm{p}<0.05$; RBTL when the target levels of SBP are reached $-0.87 \pm 0.03$, and when the SBP target levels are not reached $-0.84 \pm 0.08$, $\mathrm{p}>0.05$; RLTL when the target levels of DBP are reached $-1.08 \pm 0.04$, and when the target levels of DBP are reached $-0.90 \pm 0.07, \mathrm{p}<0.05$; RBTL when reaching target DBP levels $-0.85 \pm 0.04$, if not reaching target DBP levels $-0.83 \pm 0.05$, $\mathrm{p}>0.05$, respectively). Thus, there is a unidirectional tendency for shortening RLTL and RBTL when the target levels of SBP and DBP are not reached.
When carrying out a similar analysis of carbohydrate metabolism among group 2 patients: the RTL was assessed in the carbohydrate metabolism compensation and in its absence, no convincing data was obtained on the effect of glycemic control on the RTL (Tab. 3).

A longer RTL was observed in the buccal epithelium cells, while in the blood leukocytes, on the contrary, there was a shortening of the RTL at the levels of fasting glucose and glycosylated hemoglobin close to normal. In the subanalysis of the impact of achieving the target SBP and DBP, an increase in the RTL occurred, regardless of the level of glycemic control.

To determine the effect of $\mathrm{BP}$ and carbohydrate indicators changing in two directions: reaching the target levels and not reaching them, on the RTL, the variance analysis was used according to the traditional scheme. Performing the variance analysis in group 1 revealed a significant effect of DBP $(p=0.046)$ on the distribution of the RBTL by quartiles. Also in group 1, a reliably significant effect of reaching the target SBP levels on the presence of a longer RLTL $(\mathrm{p}=0.001)$ was revealed. The dispersion analysis in group 2 revealed the presence of a reliably significant effect of the level of fasting glucose $(p=0.029)$ on the RBTL. The significant effect of the level of glycosylated hemoglobin $(p=0.034)$ on the distribution of the RBTL by quartiles in this group was revealed. 


\section{Discussion}

The presence of significant differences in telomere length between the studied groups confirms the existing opinion about the more pronounced synergistic negative effect of age-associated comorbidity on the aging process than isolated pathology. The presence of shortened telomeres in hypertensive patients compared with controls in our study is quite consistent with the data of other authors $[2,6]$. The absence of a significant effect of achieving target BP levels on changes in the RTL in patients with isolated hypertension can be explained by the fact that hypertensive subjects already experienced excessive telomere depletion at the early stages of the disease (the average duration of hypertension in our study was $10.2 \pm 3.7$ years), which makes an additional change in telomere length with relative BP control less obvious. However, this phenomenon requires further study.

It is known that the achievement of target BP levels in T2DM is no less important for predicting the further course of the disease and the development of its complications than the achievement of the target level of glycemic control [16]. The presence of concomitant T2DM in group 2 in our study is accompanied by a significant shortening of the RTL when the target levels of both SBP and DBP are not reached, which is a significant fact confirming the importance of BP control in diabetes. The unidirectional tendency to shorten both RLTL and RBTL when target levels of BP are not reached can also serve as evidence of the special importance of BP control in the presence of concomitant T2DM.

The absence of the significant effect of carbohydrate metabolism compensation in diabetes on the RTL in our study can possibly be explained by the absence of diabetic complications in our patients according to the study design, as well as metformin use, which has a beneficial effect not only on carbohydrate metabolism, but also reduces the manifestation of oxidative stress which can lead to inhibition of vascular aging progression $[17,18]$.

All this indicates the necessity for a more detailed study of this issue, taking into account not only the achievement of target levels of studied factors but also the features of pharmacological and nonpharmacological methods of exposure. Our data are in support of higher plasticity of shortening and elongations of RTL, including under the influence of BP levels [19]. In addition, it is necessary to take into account TL interindividual variability.

The study has several limitations. The inconsistency of our work data is also explained by the fact that we studied the length of telomeres in different tissue samples (leukocytes and buccal epithelium cells), which are subjected to various influences, including external ones. The sample size in this study does not allow for a more complete analysis, which would include an assessment of different patient habits, physical activity, individual variability of possible drug effects on telomere attrition, etc.

\section{Conclusions}

In patients with isolated hypertension and hypertension in combination with type 2 diabetes mellitus, the reduction in the relative telomere length compared to normotensive individuals was observed, and in patients with comorbid pathology, the changes were more pronounced. In case of isolated hypertension, the achievement of the blood level targets associated with a controversial effect on relative telomere length both in blood leukocytes and in the buccal epithelium. Achieving the target values of blood pressure has a more significant effect on the relative telomere length than the glycemic control in patients with combined course of hypertension and type 2 diabetes mellitus. Additional determining the relative telomere length in buccal epithelial cells in combination with determining the relative telomere length of blood leukocytes enhances the diagnostic and prognostic power of this parameter when assessing the efficacy of correcting cardiovascular risk factors such as blood pressure.

The article is a fragment of the research project 'Improving the diagnosis and treatment of comorbid pathology (arterial hypertension and type 2 diabetes mellitus) based on the assessment of cardiac hemodynamics, metabolism and pharmacogenetic trial', state registration No $0118 \mathrm{U} 000923$.

\section{References}

1. Rizvi S, Raza ST, Mahdi F. Telomere length variations in aging and age-related diseases. Curr Aging Sci. 2014; 7(3): 161-167, indexed in Pubmed: 25612739.

2. Bhupatiraju C, Saini D, Patkar S, et al. Association of shorter telomere length with essential hypertension in Indian population. Am J Hum Biol. 2012; 24(4): 573-578, doi: 10.1002/ajhb.22264, indexed in Pubmed: 22431479.

3. Arsenis NC, You T, Ogawa EF, et al. Physical activity and telomere length: Impact of aging and potential mechanisms of action. Oncotarget. 2017; 8(27): 45008-45019, doi: 10.18632/oncotarget.16726, indexed in Pubmed: 28410238.

4. Harte AL, da Silva NF, Miller MA, et al. Telomere length attrition, a marker of biological senescence, is inversely correlated with triglycerides and cholesterol in South Asian males with type 2 diabetes mellitus. Exp Diabetes Res. 2012; 2012: 895185, doi: 10.1155/2012/895185, indexed in Pubmed: 22474429. 
5. Ma D, Zhu W, Hu S, et al. Association between oxidative stress and telomere length in Type 1 and Type 2 diabetic patients. J Endocrinol Invest. 2013; 36(11): 1032-1037, doi: 10.3275/9036, indexed in Pubmed: 23873360.

6. Yang Z, Huang X, Jiang H, et al. Short telomeres and prognosis of hypertension in a chinese population. Hypertension. 2009; 53(4): 639-645, doi: 10.1161/HYPERTENSIONAHA.108.123752, indexed in Pubmed: 19255364.

7. Fyhrquist F, Saijonmaa O, Strandberg T. The roles of senescence and telomere shortening in cardiovascular disease. Nat Rev Cardiol. 2013; 10(5): 274-283, doi: 10.1038/nrcardio.2013.30, indexed in Pubmed: 23478256.

8. Kolyada AK, Vayserman AM, Krasnenkov DS, et al. Issledovanie dlinyi telomer u patsientov s boleznyu Parkinsona [Study of telomere length in patients with Parkinson's disease]. Zhurnal nevrologii i psihiatrii. 2014; 114(8): 59-62, indexed in Pubmed: 25345632.

9. Gadalla SM, Cawthon R, Giri N, et al. Telomere length in blood, buccal cells, and fibroblasts from patients with inherited bone marrow failure syndromes. Aging (Albany NY). 2010; 2(11): 867-874, doi: 10.18632/aging. 100235, indexed in Pubmed: 21113082.

10. Krasnenkov DS, Loseva DA, Kolyada AK, et al. Dlina telomer v bukkalnom epitelii u zhiteley raznogo vozrasta kievskoy oblasti [Telomere length in the buccal epithelium of the Kiev region residents of different ages]. Probl. Stareniya i Dolgoletiya. 2015; 24(2): 111-119.

11. Kjeldsen SE, Aksnes TA, Ruilope LM. Clinical implications of the 2013 ESH/ESC hypertension guidelines: targets, choice of therapy, and blood pressure monitoring. Drugs R D. 2014; 14(2): 31-43, doi: 10.1007/s40268-014-0049-5, indexed in Pubmed: 24842751.

12. Inzucchi SE, Bergenstal RM, Buse JB, et al. Management of hyperglycemia in type 2 diabetes, 2015: a patient-centered approach: update to a position statement of the American Diabetes Association and the European Association for the Study of Diabetes. Diabetes Care. 2015; 38(1): 140-149, doi: 10.2337/dc14-2441, indexed in Pubmed: 25538310.
13. Cawthon RM. Telomere length measurement by a novel monochrome multiplex quantitative PCR method. Nucleic Acids Res. 2009; 37(3): e21, doi: 10.1093/nar/gkn1027, indexed in Pubmed: 19129229.

14. Weischer M, Bojesen SE, Cawthon RM, et al. Short telomere length, myocardial infarction, ischemic heart disease, and early death. Arterioscler Thromb Vasc Biol. 2012; 32(3): 822-829, doi: 10.1161/ ATVBAHA.111.237271, indexed in Pubmed: 22199369.

15. Williams B, Mancia G, Spiering W, et al. Authors/Task Force Members:. 2018 ESC/ESH Guidelines for the management of arterial hypertension: The Task Force for the management of arterial hypertension of the European Society of Cardiology and the European Society of Hypertension: The Task Force for the management of arterial hypertension of the European Society of Cardiology and the European Society of Hypertension. J Hypertens. 2018; 36(10): 1953-2041, doi: 10.1097/HJH.0000000000001940, indexed in Pubmed: 30234752.

16. Ermakova EA, Ametov AS, Chernikova NA. Saharnyiy diabet i arterialnaya gipertenziya [Diabetes mellitus and arterial hypertension]. Arterialnaya Gipertenziya. 2015; 12: 12-17.

17. Esteghamati A, Eskandari D, Mirmiranpour H, et al. Effects of metformin on markers of oxidative stress and antioxidant reserve in patients with newly diagnosed type 2 diabetes: a randomized clinical trial. Clin Nutr. 2013; 32(2): 179-185, doi: 10.1016/j. clnu.2012.08.006, indexed in Pubmed: 22963881.

18. Lodovici M, Bigagli E, Luceri C, et al. Gender-related drug effect on several markers of oxidation stress in diabetes patients with and without complications. Eur J Pharmacol. 2015; 766: 86-90, doi: 10.1016/j.ejphar.2015.09.041, indexed in Pubmed: 26424110.

19. Raschenberger J, Kollerits B, Titze S, et al. GCKD study Investigators. Do telomeres have a higher plasticity than thought? Results from the German Chronic Kidney Disease (GCKD) study as a high-risk population. Exp Gerontol. 2015; 72: 162-166, doi: 10.1016/j. exger.2015.09.019, indexed in Pubmed: 26423240. 